\title{
INAUGURATION
}

\section{Opening addresses}

I am very pleased to see that so many delegates from all over the world have come to Würzburg to take part in our Symposium on leprosy treatment and I would like to warmly welcome you all to our city.

To my understanding this Symposium will be an opportunity to present and to discuss recent results of chemotherapy research and related fields in an unconstrained but nevertheless very concentrated atmosphere.

I am very gratified to see that scientists who are supported by the German Leprosy Relief Association will have the chance to show that the German Leprosy Relief Association has been interested in finding effective medicines for a long time. It was above all Professor Joachim Seydel, Head of the Department of Chemotherapy Research and Head of the so-called 'Leprosy Research Crew' at the Institute of Experimental Biology and Medicine at Borstel, who by his worldwide connections with many of our lecturers decisively contributed to the shaping of our programme.

I very much hope that besides making a detailed inventory for which Session I 'Clinical Aspects' shall be especially useful, we will also be able to present and discuss the latest techniques and results from renowned scientific institutes.

Emphasis will be laid on the fact that completely different ways may lead to the same goal, that is effective control of chemotherapy; Session II therefore has the heading 'Test Models for the Effective Control of Chemotherapy'. Imagination will take the floor in Session III when 'Developments and Future Aspects' will be discussed.

Enough time will be left over between the Sessions to have personal talks and to become acquainted with each other, if this should still be necessary in this circle. It will no doubt be necessary to establish contacts between, well I may say so, laboratory scientists and field scientists who should discuss their individual needs and requirements.

We also wish, however to get to know scientists from the University of Würzburg who direct or work in institutes which have or could have connections with leprosy work. We hope to be able to make the first step here towards closer co-operation between the German Leprosy Relief Association and the University of Würzburg. For that reason I am very pleased that one of the members of the University of Würzburg, who is also one of our Medical Advisors, namely Dr Klaus Fleischer, will give the keynote address today.

We are above all very thankful, Mr Vice President, to the University for giving us the chance to have our Symposium here in these rooms. 
Apart from encouraging you to use the following three days for the benefit of scientific matters of leprosy therapy and of the patients suffering from this disease, I would also urge you not to forget to enjoy the beauties of our city, the Lord Mayor of which I cordially welcome here. The GLRA members of staff will be pleased to do their very best to make you feel comfortable and please do not hesitate to contact Frau Rößler, Frau Hajek or me, if there is any problem.

Finally I wish all of us three successful and pleasant days.

Thank you so much! 